\title{
Elevated C-reactive protein increases diagnostic accuracy of algorithm-defined Stroke Associated Pneumonia in afebrile patients
}

DOI:

10.1177/1747493018798527

\section{Document Version}

Accepted author manuscript

Link to publication record in Manchester Research Explorer

Citation for published version (APA):

Kalra, L., Smith, C., Hodsoll, J., Vail, A., Irshad, S., \& Manawadu, D. (2018). Elevated C-reactive protein increases diagnostic accuracy of algorithm-defined Stroke Associated Pneumonia in afebrile patients. International Journal of Stroke. https://doi.org/10.1177/1747493018798527

\section{Published in:}

International Journal of Stroke

\section{Citing this paper}

Please note that where the full-text provided on Manchester Research Explorer is the Author Accepted Manuscript or Proof version this may differ from the final Published version. If citing, it is advised that you check and use the publisher's definitive version.

\section{General rights}

Copyright and moral rights for the publications made accessible in the Research Explorer are retained by the authors and/or other copyright owners and it is a condition of accessing publications that users recognise and abide by the legal requirements associated with these rights.

\section{Takedown policy}

If you believe that this document breaches copyright please refer to the University of Manchester's Takedown Procedures [http://man.ac.uk/04Y6Bo] or contact uml.scholarlycommunications@manchester.ac.uk providing relevant details, so we can investigate your claim.

\section{OPEN ACCESS}




\section{Elevated C-reactive protein increases diagnostic accuracy of algorithm-defined Stroke}

Associated Pneumonia in afebrile patients

Lalit Kalra PhD*, ${ }^{1}$ Craig J. Smith MD*, John Hodsoll PhD, ${ }^{3}$ Andy Vail MSc, ${ }^{4}$ Saddif Irshad MSc, ${ }^{1}$ Dulka Manawadu MRCP,${ }^{5}$ on behalf of the STROKE-INF Investigators

1. Department of Basic and Clinical Neurosciences, Institute of Psychiatry, Psychology and Neurosciences, King's College London, London SE5 8AF, UK

2. Greater Manchester Comprehensive Stroke Centre and Division of Cardiovascular Sciences, University of Manchester, Manchester Academic Health Science Centre, Salford Royal NHS Foundation Trust, Salford M6 8HD, UK

3. Biostatistics Department, NIHR Biomedical Research Centre for Mental Health and Institute of Psychiatry, Psychology and Neurosciences, King’s College London, London SE5 8AF, UK

4. Centre for Biostatistics, University of Manchester, Manchester M13 9PL, UK

5. Department of Clinical Neurosciences, King's College Hospital NHS Foundation Trust, London SE5 8RS, UK

*These authors contributed equally to the manuscript.

Key words: acute stroke, post-stroke pneumonia, diagnostic accuracy, C-reactive protein Word count: Paper: 3789 Abstract: 246

References: 17

Tables: 2

Figures: 1

\section{Corresponding Author:}

Lalit Kalra, Department of Basic and Clinical Neurosciences, PO Box 41, Institute of Psychiatry, Psychology and Neurosciences, King's College London, London SE5 8AF, UK. Telephone +44 (0) 203299 3487; e-mail: lalit.kalra@ kcl.ac.uk 


\section{ABSTRACT}

Background and aim: Pyrexia dependent clinical algorithms may under or over diagnose Stroke Associated Pneumonia (SAP). This study investigates whether inclusion of elevated C-reactive protein (CRP) as a criterion improves diagnosis.

Methods: The contribution of CRP $\geq 30 \mathrm{mg} / \mathrm{L}$ as an additional criterion to a Centres for Disease Control and Prevention (CDC) based algorithm incorporating pyrexia with chest signs and leucocytosis and/or chest infiltrates to diagnose SAP was assessed in 1088 acute stroke patients from 37 UK stroke units. The sensitivity, specificity and positive predictive value of different approaches were assessed using adjudicated SAP as the reference standard. Results: Adding elevated CRP to all algorithm criteria did not increase diagnostic accuracy compared with the algorithm alone against adjudicated SAP (sensitivity 0.74 [95\% CI 0.65 0.81] v 0.72 [95\% CI 0.64-0.80], specificity 0.97 [95\% CI 0.96-0.98] for both; kappa (0.70 [95\% C.I. 0.63-0.77] for both). In afebrile patients ( $\mathrm{n}=965)$, elevated CRP with chest and laboratory findings had sensitivity of 0.84 [95\% CI $0.67-0.93]$, specificity of 0.99 [95\% CI 0.98-1.00] and kappa 0.80 [95\% C.I. 0.70-0.90]). The modified algorithm of pyrexia or elevated CRP and chest signs with infiltrates or leucocytosis had sensitivity of 0.94 [95\% CI 0.87-0.97], specificity of 0.96 [95\% CI 0.94-0.97] and kappa of 0.88 [95\% C.I. 0.84-0.93]) against adjudicated SAP.

Conclusions: An algorithm consisting of pyrexia or CRP $\geq 30 \mathrm{mg} / \mathrm{L}$, positive chest signs, leucocytosis and/or chest infiltrates has high accuracy and can be used to standardise SAP diagnosis in clinical or research settings.

Trial Registration: http://www.isrctn.com/ISRCTN37118456 


\section{INTRODUCTION}

Stroke Associated Pneumonia (SAP) has a significant effect on stroke outcome, being independently associated with nearly one-third of all deaths and increasing the likelihood of dependence at discharge. $[1,2]$ Many studies have shown the difficulties in diagnosing stroke associated pneumonia (SAP) because of the heterogeneity in the criteria used and lack of prospective studies validating diagnostic criteria.[3] To confound matters, SAP is often used as a convenient diagnosis in severely-ill or terminal stroke patients, many of whom do not fulfil the criteria for diagnosis.[4] The consequences of the lack of a consistent approach for diagnosing SAP include inappropriate use of antibiotics in practice and inconclusive research findings.[3] In order to reduce the variations in the diagnosis of SAP, the international multidisciplinary Pneumonia In Stroke ConsEnsuS (PISCES) Group proposed consistent use of algorithmic criteria, because of their objectivity and applicability to both clinical and research settings.[5]

A recent study of the diagnostic utility of a modified version of the Centres for Disease Control and Prevention (CDC) criteria for hospital acquired pneumonia [6] showed that although algorithmic approaches were very good at excluding patients who do not have SAP (high specificity), they were limited in predicting those with SAP (low sensitivity).[7] This is because pyrexia is a component of the modified CDC algorithm criteria,[6] and it is well known that many elderly stroke patients do not develop fever despite having pneumonia.[8] In the study, $25 \%$ of patients adjudicated to have SAP were missed by the algorithm despite many having new chest signs, infiltrates on radiography and/or leucocytosis because they were afebrile.[7] Furthermore, the thresholds for fever used in diagnostic algorithms are arbitrary, and have not been validated.[5] 
C-reactive protein (CRP) is a widely used acute-phase biomarker in clinical practice, and elevated levels have been shown to be predictive of infections in stroke patients.[9,10] A study in older people showed that elevated CRP values were highly sensitive for the diagnosis of a bacterial infection, even in the absence of pyrexia.[11] However, mild elevations of CRP are common in acute stroke patients, even in the absence of infections, because of inflammation associated with infarction or haemorrhage.[12] Indeed, the precise role of CRP in the diagnostic approach to SAP is uncertain based on a recent systematic review and consensus statement, [5] and analysis of the Intravascular Cooling Treatment in Acute Stroke-2 (ICTuS-2) Trial. [13] A more recent study using receiver operating characteristics analysis showed that CRP concentration $>25.60 \mathrm{mg} / \mathrm{L}$ was the optimal diagnostic threshold for the diagnosis of SAP, with high sensitivity (0.85) but low specificity (0.67).[8]

As the pyrexia based CDC algorithm has high specificity but low sensitivity [7] and the CRP threshold has high sensitivity but low specificity for diagnosis of SAP,[8] we hypothesised that combining a CRP threshold criterion with the pyrexia based algorithm would have high specificity and high sensitivity for SAP diagnosis. The objective of this study was therefore to determine if incorporating $\mathrm{CRP} \geq 30 \mathrm{mg} / \mathrm{L}$ as an additional criterion to the existing $\mathrm{CDC}$ based algorithm $[7,14]$ would improve accuracy of SAP diagnosis using adjudicated SAP as the reference standard. 


\section{METHODS}

The STROKE-INF study was a prospective, multicentre, cluster randomised, open-label controlled trial with blinded end-point assessment, undertaken in 1217 stroke patients with dysphagia (nil by mouth) recruited from 37 UK stroke units .[14] Patients were eligible for inclusion if they were aged over 18 years, had a confirmed diagnosis of new stroke, onset of symptoms $\leq 48$ hours at recruitment and were considered unsafe to swallow because of impaired consciousness or failed bedside swallowing assessment.[15] Patients with preexisting dysphagia, pyrexia or known infection at admission, antibiotics within last 7 days, or expected survival $<14$ days were excluded. The primary outcome was SAP in the first 14 days, assessed using a CDC criteria-based, hierarchical algorithm masked to allocation. Written informed consent/assent was provided by patients or the next of kin. The study (ISRCTN 37118456) was approved by the National Research Ethics Committee (08/H0803/1).

Patient characteristics were recorded on enrolment. Data on respiratory rate, temperature, chest symptoms and signs, white cell counts and CRP were collected prospectively at baseline, 2, 4, 7, 10 and 14 days. Local guidelines for radiological and microbiological investigations were followed for suspected SAP. The algorithmic SAP diagnosis was based on CDC criteria for pneumonia [6] and performed at the end of trial by the study statistician (JH) who was masked to physician diagnosis, treatment details or outcomes and independent of treating physicians at trial centres or the central trial team. Eight clinical/laboratory observations at 6 time-points were interrogated using Stata software (StataCorp. 2009. Stata Statistical Software: Release 11. College Station, TX) as yes/no responses for: 
1) Temperature $\geq 37 \cdot 5^{\circ} \mathrm{C}$ on two consecutive measurements or a single measurement of $\geq 38 \cdot 0^{\circ} \mathrm{C}(\mathrm{C} 1)$ AND

2) Respiratory rate $\geq 20 / \mathrm{min}$ OR cough and breathlessness OR purulent sputum (C2) AND

3) White cell count $>11.0 \times 10^{9} / \mathrm{L}$ OR new chest infiltrates on X-ray OR positive sputum culture/microbiology OR positive blood culture (C3).

A diagnosis of SAP or no SAP on the algorithm could not be established in 129 (10\%) patients because of missing data (Fig 1). Analyses of diagnostic accuracy were undertaken in 1088 patients; there were no differences in the baseline characteristics or outcomes between the 1217 patients in the STROKE-INF trial and the 1088 patients in the analysis, which could result in selection bias (Table 1).

Components of the algorithmic criteria, clinical findings and all laboratory investigations for the whole episode of hospitalisation of patients (including detailed chest radiology reports and free-text clinical narrative) were reviewed by two independent expert adjudicators (a chest physician and a general physician not involved with the study). The adjudicators worked separately for reassignment as SAP or no SAP, and were unaware of diagnoses made by treating physicians in trial centres or of algorithm diagnosis made by the statistician. In the event of disagreement, clinical data were reviewed jointly by the two experts and consensus reached on assignment. Adjudicated SAP was used as the reference standard for diagnosis of SAP using the post-hoc algorithm approaches applied blindly to trial data.

All analyses were pre-specified, and undertaken by researchers masked to the findings of the original STROKE-INF study. In the first analysis, performance of the algorithm $(\mathrm{C} 1+\mathrm{C} 2+\mathrm{C} 3)$ 
to diagnose SAP was compared with approaches that used: 1$)$ the algorithm $(\mathrm{C} 1+\mathrm{C} 2+\mathrm{C} 3)$ AND elevated CRP $\geq 30 \mathrm{mg} / \mathrm{L}$ at any of the six time-points (to reduce false positive rate of the algorithm), and 2) the algorithm $(\mathrm{C} 1+\mathrm{C} 2+\mathrm{C} 3) \mathrm{OR} \mathrm{C} 2+\mathrm{C} 3$ with elevated $\mathrm{CRP} \geq 30 \mathrm{mg} / \mathrm{L}$ at any of the six time-points (to reduce false negative rate) as additional criteria for diagnosis. In the second analysis, the CDC criterion of temperature (C1) was excluded as a criteria and substituted by elevated CRP at any one of the 6 measurement time points, and SAP diagnosed if this and $\mathrm{C} 2+\mathrm{C} 3$ criteria were met for the whole patient group and for the subset of afebrile patients $(n=965)$. Diagnostic accuracy was assessed by calculating the sensitivity, specificity, positive predictive value, negative predictive value, false-positive and false-negative rates and compared using McNemar's test. Agreement of these approaches using CRP to diagnose SAP with adjudicated SAP was assessed using Cohen's kappa statistic. 


\section{RESULTS}

The baseline characteristics of the patients included in this study are given in Table 1.

Table 1: Baseline Patient Demographics

\begin{tabular}{|c|c|c|c|}
\hline & $\begin{array}{l}\text { All patients in the } \\
\text { STROKE-INF Trial }\end{array}$ & $\begin{array}{c}\text { Patients with } \\
\text { algorithm SAP } \\
\text { diagnosis }\end{array}$ & Afebrile patients \\
\hline No of patients & 1217 & $1088(89.4 \%)$ & $965(79.3 \%)$ \\
\hline Age: mean (SD) & $77 \cdot 9(12.1)$ & $77.10(12.25)$ & 77.13 (12.44) \\
\hline (missing) & 1 & 0 & 0 \\
\hline Male: n(\%) & $523(43 \cdot 0 \%)$ & 466 (42.8\%) & $398(41.2 \%)$ \\
\hline (missing) & 4 & 4 & 3 \\
\hline \multicolumn{4}{|l|}{ Stroke Type: n(\%) } \\
\hline Ischaemic & $1091(89 \cdot 7 \%)$ & 973 (89.5\%) & 874 (90.1\%) \\
\hline Haemorrhagic & $125(10 \cdot 2 \%)$ & $114(10.5 \%)$ & 91 (9.9\%) \\
\hline (missing) & 1 & 1 & 0 \\
\hline Thrombolysis: $n(\%)$ & 397 (32.7\%) & 347 (31.9\%) & $304(31.5 \%)$ \\
\hline (missing) & 3 & 0 & 0 \\
\hline NIHSS: median (IQR) & $15(9-20)$ & $15(9-20)$ & $15(8-20)$ \\
\hline (missing) & 4 & 4 & 0 \\
\hline \multicolumn{4}{|l|}{ Co- morbidities: $n(\%)$} \\
\hline Hypertension & $837(68.9 \%)$ & 751 (69.1\%) & $660(68.4 \%)$ \\
\hline Diabetes & $202(16.6 \%)$ & 179 (16.5\%) & $158(16.4 \%)$ \\
\hline Atrial Fibrillation & $448(36 \cdot 9 \%)$ & 393 (36.1\%) & $339(35.1 \%)$ \\
\hline Previous Strokes & $352(29.0 \%)$ & $316(29.0 \%)$ & $282(29.2 \%)$ \\
\hline (missing) & 1 & 0 & 0 \\
\hline Pre-morbid mRS (0-2): n(\%) & $988(81.3 \%)$ & 939 (86.3\%) & $799(82.8 \%)$ \\
\hline (missing) & $18(1.5 \%)$ & $9(0.8 \%)$ & $6(0.6 \%)$ \\
\hline Mortality at 90 days: $n(\%)$ & $342(28.1 \%)$ & $231(21.2 \%)$ & $256(26.5 \%)$ \\
\hline (missing) & $36(3.0 \%)$ & 0 & 0 \\
\hline mRS $0-2$ at 90 days: $n(\%)$ & $230(18.9 \%)$ & 274 (25.9\%) & $201(20.8 \%)$ \\
\hline (missing) & $36(3.0 \%)$ & $29(2.7 \%)$ & $28(2.9 \%)$ \\
\hline
\end{tabular}


The age, sex, vascular risk factors, pre-morbid function or stroke severity characteristics of afebrile patients $(n=965)$ were comparable to the whole patient group in whom algorithm diagnosis of SAP was possible (1088) and not different from all patients participating in the STROKE-INF study.

Adjudicated SAP was present in 129/1088 (11.8\%) patients. Of these, 97 (75\%) had pyrexia (C1), $48(37 \%)$ had new infiltrates on a chest radiograph (C3) and $39(30 \%)$ had productive cough (C2). SAP was diagnosed by the algorithm in in $123(11.3 \%)$ patients. The algorithm had high specificity (0.97), low sensitivity (0.72) and moderate agreement (kappa 0.70) against adjudicated SAP (Table 2). The algorithm missed SAP in 37 patients because the fever criterion was not met (false negative). On the other hand, 30 patients without focal chest findings met algorithm criteria for SAP (false positive) because of fever, leucocytosis and tachypnoea.

The inclusion of elevated CRP as an additional criterion to the full algorithm did not increase its overall diagnostic accuracy or agreement with adjudicated SAP (Table 2). However, if the requirements for SAP diagnosis included patients who fulfilled algorithmic criteria or had elevated CRP with definitive chest findings regardless of pyrexia, diagnostic accuracy improved significantly to reach sensitivity of 0.94 , specificity of 0.96 and agreement with adjudicated SAP of nearly 0.90 (Table 2 ).

It was possible that both pyrexia and CRP, being components of the acute phase response were measuring the same phenomenon related to infection, and elevated CRP may be a better biomarker of infection compared with temperature. In the second analysis, the temperature criterion of the $\mathrm{CDC}$ based algorithm was substituted by $\mathrm{CRP} \geq 30 \mathrm{mg} / \mathrm{L}$ in the presence of 
Table 2: Accuracy of algorithm AND/OR elevated CRP in the diagnosis of Stroke Associated Pneumonia (SAP) using adjudicated SAP as the reference standard in 1088 stroke patients

\begin{tabular}{|c|c|c|c|c|c|c|}
\hline & \multicolumn{2}{|c|}{ Algorithm (C1+C2+C3) } & \multicolumn{2}{|c|}{$\begin{array}{c}\text { Algorithm (C1+C2+C3) AND } \\
\text { CRP }\end{array}$} & \multicolumn{2}{|c|}{$\begin{array}{c}\text { Algorithm (C1+C2+C3) OR } \\
\mathrm{CRP}+\mathrm{C} 2+\mathrm{C} 3\end{array}$} \\
\hline & & $95 \%$ & & $95 \%$ & & $95 \%$ \\
\hline & & Confidence Interval & & Confidence Interval & & Confidence Interval \\
\hline SAP Prevalence & $11.9 \%$ & $10.0 \%-13.9 \%$ & $11.6 \%$ & $10.0 \%-14.0 \%$ & $11.9 \%$ & $10.1 \%-14.1 \%$ \\
\hline Sensitivity & 0.72 & $0.64-0.80$ & 0.74 & $0.65-0.81$ & 0.94 & $0.88-0.97$ \\
\hline Specificity & 0.97 & $0.96-0.98$ & 0.97 & $0.95-0.98$ & 0.96 & $0.94-0.97$ \\
\hline False Positive Rate & $24.4 \%$ & & $25.8 \%$ & & $14.8 \%$ & \\
\hline False Negative Rate & $3.7 \%$ & & $3.5 \%$ & & $0.9 \%$ & \\
\hline Positive Predictive Value & 0.76 & $0.67-0.83$ & 0.75 & $0.66-0.81$ & 0.75 & $0.68-0.82$ \\
\hline Negative Predictive Value & 0.96 & $0.95-0.98$ & 0.96 & $0.95-0.98$ & 0.99 & $0.98-1.00$ \\
\hline Positive Likelihood Ratio & 23.05 & $15.95-33.30$ & 21.4 & $15.07-30.39$ & 23.05 & $16.9-31.44$ \\
\hline Negative Likelihood Ratio & 0.29 & $0.22-0.38$ & 0.27 & $0.20-0.36$ & 0.06 & $0.03-0.12$ \\
\hline
\end{tabular}

Agreement

Algorithm $(\mathrm{C} 1+\mathrm{C} 2+\mathrm{C} 3)$ v adjudicated SAP: kappa 0.70 [95\% C.I. 0.63-0.78]

Algorithm (C1+C2+C3) AND CRP v adjudicated SAP: kappa 0.70 [95\% C.I. 0.63-0.77]

Algorithm (C2+C3) OR CRP + C2+C3 v adjudicated SAP: kappa 0.88 [95\% C.I. 0.84-0.93]

Temperature (C1): Temperature $\geq 37 \cdot 5^{\circ} \mathrm{C}$ on two consecutive measurements or a single measurement of $\geq 38 \cdot 0^{\circ} \mathrm{C}$

Respiratory (C2): Tachypnoea (Respiratory Rate $\geq 20 / \mathrm{min}$ ); Cough or dyspnoea: New onset or worsening; Increased secretions: Purulent sputum, or increased respiratory secretions, or increased suctioning requirements

Laboratory (C3): Leucopoenia ( $<4000 \mathrm{WBC} / \mathrm{mm} 3$ ) or leucocytosis ( $>11,000 \mathrm{WBC} / \mathrm{mm} 3)$; At least 1 chest radiograph with new infiltrate or consolidation; a positive microbiology results with identification of causative organism; a positive blood culture which identifies a pulmonary pathogen

Algorithm criteria for SAP: temperature criteria (C1) + at least 1 respiratory criterion (C2) + at least 1 laboratory criterion (C3) 
other chest and laboratory criteria, and applied to the whole dataset and the subset of afebrile patients. The second analysis showed that this improved the diagnostic accuracy of the algorithm with sensitivity of 0.80 , specificity of 0.98 , positive predictive value of 0.78 and agreement with adjudicated SAP of 0.80, especially in afebrile patients (Supplementary Table). Elevated CRP also appeared to be associated with higher mortality in afebrile patients with adjudicated SAP. CRP levels were elevated in 30/36 (83.3\%) apyrexial SAP patients, 19 $(63.3 \%)$ of whom died. 


\section{DISCUSSION}

Our study shows that adding CRP to the full algorithm in all patients did not increase diagnostic performance. However, $\mathrm{CRP} \geq 30 \mathrm{mg} / \mathrm{L}$, in the presence of chest findings and leucocytosis or new radiological chest infiltrates, is highly suggestive of SAP in afebrile stroke patients and has very good agreement (kappa 0.80) with adjudicated diagnosis of SAP. The greatest diagnostic accuracy (sensitivity 0.94 , specificity 0.96 , kappa 0.88 ), for the whole patient cohort (regardless of pyrexia) was achieved using the criteria:

1) Temperature $\geq 37 \cdot 5^{\circ} \mathrm{C}$ on two consecutive measurements or a single measurement of $\geq 38 \cdot 0^{\circ} \mathrm{C}, \mathrm{OR}$, a single measurement $\mathrm{CRP} \geq 30 \mathrm{mg} / \mathrm{L}, \mathrm{AND}$

2) Respiratory rate $\geq 20$ / min, OR, cough and breathlessness, OR, purulent sputum, AND

3) White cell count $>11.0 \times 10^{9} / \mathrm{L}$, OR, new chest infiltrates on X-ray, OR, positive sputum culture/microbiology OR positive blood culture.

Pyrexia, a hallmark of infection, may be absent in $25 \%$ to $66 \%$ of patients with SAP. [7, 8] Diagnosis may be facilitated by referring to blood biomarkers of inflammation, of which CRP is one of the most easily available and widely used investigations. A recent meta-analysis of 699 patients from seven studies showed higher CRP levels within 24 hours were independently associated with post-stroke infection.[16] Further, in a study of communityacquired pneumonia, CRP, but not temperature, was independently associated with extent of infiltrates on chest radiography.[17] However, CRP levels in stroke patients can be elevated due to non-infective inflammatory processes or infections elsewhere.[12] This was seen in both the STROKE-INF and the MAPS (metoclopramide to prevent pneumonia in stroke patients fed via nasogastric tubes) studies, in which $44 \%$ and $91 \%$ patients had elevated CRP 
levels but only $12 \%$ and $73 \%$ had SAP respectively.[8, 14] As most non-infective processes in stroke lead only to modest elevations in CRP, one approach would be to identify a CRP threshold above which SAP is most likely. ROC analysis of MAPS data showed that the optimal cut-off level for CRP to preserve the sensitivity required for a screening test was $25.60 \mathrm{mg} / \mathrm{L}$ but this was at the expense of lower specificity.[8] The present study combines the high sensitivity of elevated CRP and/or pyrexia for infections with the high specificity of new chest findings (whether clinical or radiological) as seen with the algorithm to develop objective criteria for identifying SAP, which have both high sensitivity and high specificity.

This study has several strengths. First, it provides external validation of the diagnostic value of a CRP threshold, derived from a smaller sample, in a large, independent, prospective dataset of actual practice collected from 37 centres across the UK. A second strength was the application of adjudicated SAP, based on assessment by two independent adjudicators, as the diagnostic reference standard. The CRP inclusive algorithm is consistent with the core criteria used in previous incidence and prevalence studies,[3] and was applied masked to adjudicated SAP, outcomes or antibiotic use. The study also has some limitations. The CRP criterion was considered to be met if any one of the 6 measurements before the endpoint of SAP was met was $\geq 30 \mathrm{mg} / \mathrm{L}$. It was not possible to correlate this with the date of onset of SAP because of timing of assessments and diagnostic rules pre-specified in the STROKEINF study. It is well-known that onset of SAP can be insidious and the time of onset and that of diagnosis may not coincide. This bias was investigated by doing further analyses requiring at least two elevated CRP values or an increase CRP value $>10 \mathrm{mg} / \mathrm{L}$ as alternative criteria but these did not show any significant changes in diagnostic accuracy. Further, whilst we were not able to use a standardised analytical approach to CRP measurement given the 
logistics of the trial, our findings reflect real-life practice with respect to measurement of CRP across a large number of UK stroke units.

Despite these limitations this is the first large study to investigate the usefulness of CRP as a potential biomarker for the diagnosis of SAP. It describes a simple algorithm incorporating CRP for diagnosis of SAP, which has high sensitivity and specificity, and could be implemented in daily practice to inform clinical decisions in stroke patients with suspicion of SAP, even in the absence of fever. Further prospective studies are required to replicate our findings and to also evaluate the utility of the diagnostic algorithm in antibiotic decision making and on relevant clinical and health-economic outcomes in stroke unit care. 


\section{Acknowledgements}

We are very grateful to the participating STROKE-INF trial centres and investigators (Supplementary material).

\section{Role of Funders}

Funded by the National Institute for Health Research (Project number PB-PG-0906-11103).

The funders had no role in the study design; collection, analysis, or interpretation of the data; or in writing of the report. The views expressed in this publication are those of the authors and not necessarily those of the NHS, NIHR or the Department of Health.

\section{Conflicts of interest}

We declare no conflicts of interest. 


\section{REFERENCES}

1. Westendorp WF, Nederkoorn PJ, Vermeij JD, et al. Post-stroke infection: a systematic review and meta-analysis. BMC Neurol 2011; 11: 110.

2. Kalra L, Hodsoll J, Irshad S, et al., STROKE-INF Investigators. Association between nasogastric tubes, pneumonia, and clinical outcomes in acute stroke patients. Neurology 2016; 87: 1352-1359.

3. Kishore AK, Vail A, Chamorro A, et al. How is pneumonia diagnosed in clinical stroke research? A systematic review and meta-analysis. Stroke 2015; 46: 1202-1209.

4. Meisel A, Smith CJ. Prevention of stroke-associated pneumonia: where next? Lancet 2015; 386: 1802-1804.

5. Smith CJ, Kishore AK, Vail A, et al. Diagnosis of stroke-associated pneumonia: recommendations from the pneumonia in stroke consensus group. Stroke 2015; 46: $2335-2340$.

6. Horan TC, Andrus M, Dudeck MA. CDC/NHSN surveillance definition of health care-associated infection and criteria for specific types of infections in the acute care setting. Am J Infect Control 2008; 36: 309-332.

7. Kalra L, Hodsoll J, Irshad S, et al., for STROKE-INF Investigators. Comparison of the diagnostic utility of physician-diagnosed with algorithm-defined stroke-associated pneumonia. J Neurol Neurosurg Psychiatry 2016; 87: 1163-1168.

8. Warusevitane A, Karunatilake D, Sim J, et al. Early diagnosis of pneumonia in severe stroke: clinical features and the diagnostic role of C-reactive protein. PLoS One 2016; 11:e0150269. 
9. Molnar T, Papp V, Banati M, et al. Relationship between C-reactive protein and early activation of leukocytes indicated by leukocyte antisedimentation rate in patients with acute cerebrovascular events. Clin Hemorheol Microcirc 2010; 44: 183-192.

10. Worthmann H, Tryc AB, Dirks M, et al. Lipopolysaccharide binding protein, interleukin-10, interleukin-6 and C-reactive protein blood levels in acute ischemic stroke patients with post-stroke infection. J Neuroinflammation 2015; 12: 13.

11. Liu A, Bui T, Van NH, et al. Serum C-reactive protein as a biomarker for early detection of bacterial infection in the older patient. Age Ageing 2010; 39: 559-565.

12. Emsley HC, Smith CJ, Gavin CM, et al. An early and sustained peripheral inflammatory response in acute ischaemic stroke: relationships with infection and atherosclerosis. J Neuroimmunol 2003; 139: 93-101.

13. Lyden P, Hemmen T, Grotta J, et al. Results of the ICTuS 2 Trial (Intravascular Cooling in the Treatment of Stroke 2). Stroke 2016; 47:2888-2895.

14. Kalra L, Irshad S, Hodsoll J, et al. Prophylactic antibiotics after acute stroke for reducing pneumonia in patients with dysphagia (STROKE-INF): a prospective, cluster-randomised, open-label, masked endpoint, controlled clinical trial. Lancet 2015; 386: 1835-1844.

15. Ramsey DJC, Smithard DG, Kalra L. Early assessments of dysphagia and aspiration risk in acute stroke patients. Stroke 2003; 34: 1252-1257.

16. Bustamante A, Vilar-Bergua A, Guettier S, et al. C-reactive protein in the detection of post-stroke infections: systematic review and individual participant data analysis. $J$ Neurochem 2017; 141: 305-314.

17. Tamayose M, Fujita J, Parrott G, et al. Correlations between extent of X-ray infiltration and levels of serum C-reactive protein in adult non-severe communityacquired pneumonia. $J$ Infect Chemother 2015; 21: 456-463. 
Fig 1. STARD diagram of participant flow for the criteria pyrexia or 个CRP + chest signs + leucocytosis or chest infiltrates

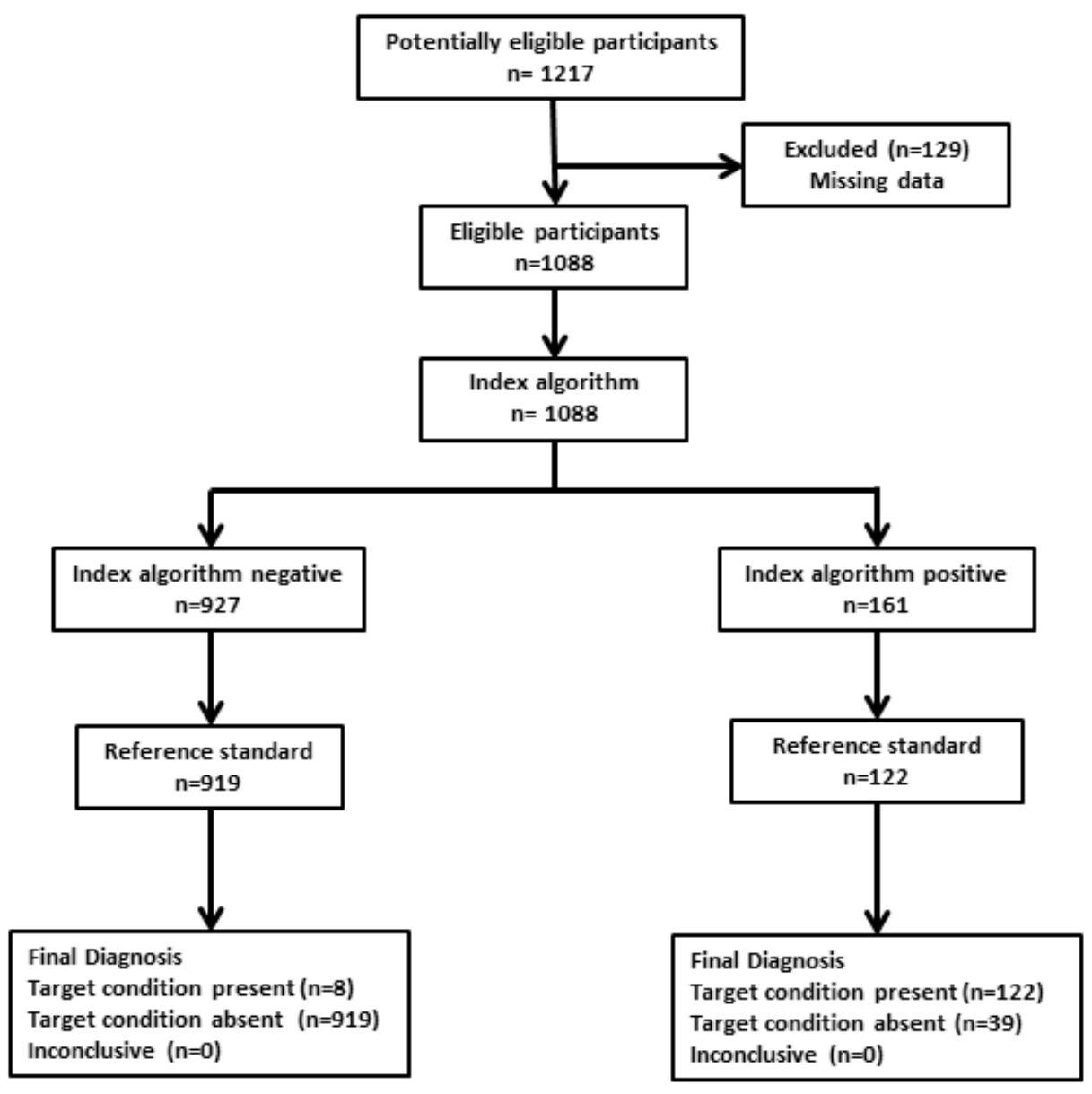


